\title{
Immunization with DNA vaccines in early life: advantages and limitations as compared to conventional vaccines
}

\author{
Claire-Anne Siegrist, Paul-Henri Lambert \\ WHO Collaborating Center for Neonatal Vaccinology, Departments of Pathology and Pediatrics, \\ University of Geneva, 1 rue Michel Servet, CH-1211 Geneva 4, Switzerland
}

\section{The need for early life immunization}

Neonatal or early infant immunizations are required to give protection from diseases caused by pathogens to which exposure occurs in early life. As an example, the median age of infants with a respiratory syncytial virus (RSV) infection severe enough to require hospitalization is below 3 months of age worldwide. A significant proportion of severe early infections are due either to encapsulated bacteria such as Haemophilus influenzae, Streptococcus pneumoniae, or Neisseria meningitidis or to intracellular agents such as viruses [RSV, measles virus (MV), rotavirus, herpes simplex viruses, cytomegalovirus], certain bacteria (Listeria monocytogenes, salmonella, mycobacteria) or other microorganisms (Chlamydiae, Ureaplasma). This enhanced susceptibility of newborns and infants to specific infectious diseases is due to limitations of both their innate and specific immune system.

\section{Immune responses in early life differ from adult responses}

The impaired function of monocytes/macrophages in newborns has been associated with reduced expression of major histocompatibility complex (MHC) class II and costimulatory molecules, decreased antigen-processing capacity and altered cytokine production. Although little is known about the development of human dendritic cells, their maturation appears to be slow and their function as antigen-presenting cells (APC) is rather poor at birth [10], thus probably contributing to the limitations of the induction of specific immune responses. Although natural killer (NK) cells are present in significant numbers at birth, their functional activity [reflected by interferon- $\gamma$ (IFN- $\gamma$ ) production and NK cell-mediated lysis] also remains low for several weeks. It is not yet known precisely which part of these limitations reflects the intrinsic immaturity of infant NK cells or their impaired activation by cytokines or factors that prevail in the 


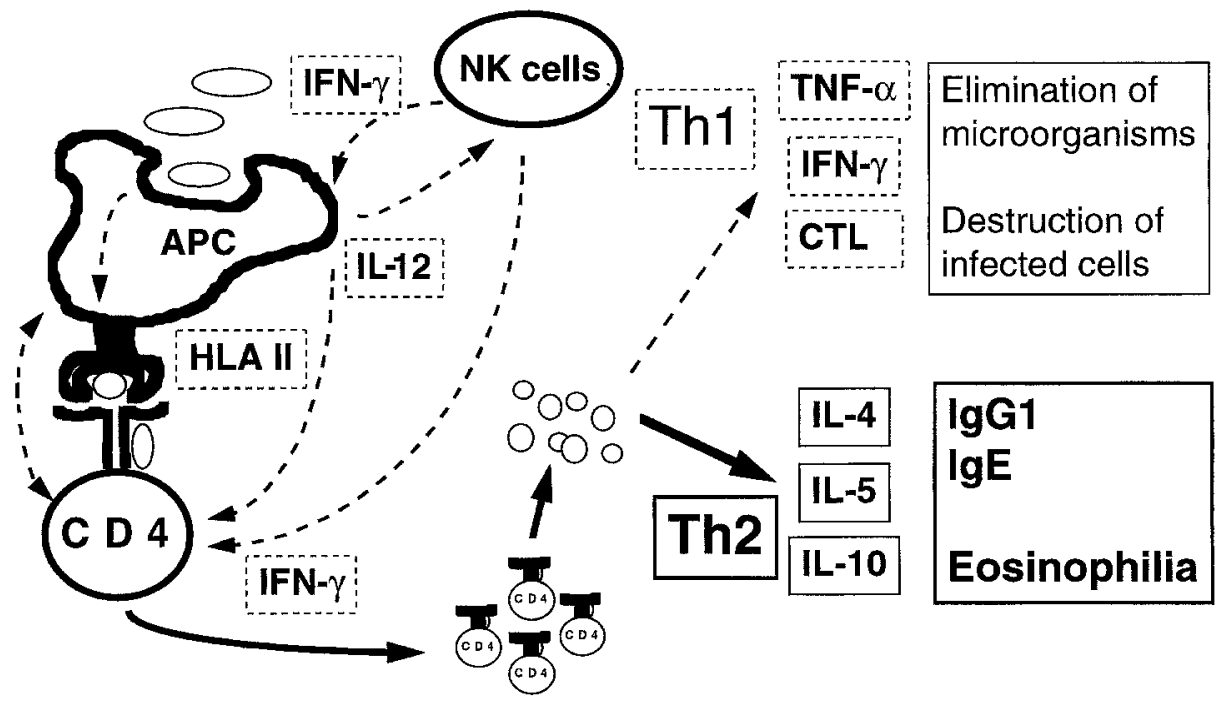

Fig. 1. Schematic representation of the preferential polarization of neonatal CD4 Th2 cell responses. Factors contributing to the preferential development of neonatal CD4 Th2 cell responses compared to adult responses have been identified at the level (i) of antigen-presenting cells $(A P C)$ : decreased antigen processing, decreased expression of major histocompatibility complex class II and co-stimulatory molecules, decreased production of cytokines such as interleukin (L)-12; (ii) of natural killer (NK) cells: decreased production of interferon- $\gamma$ (IFN- $\gamma$ ) acting both on APC and CD4 T cells; (iii) of CD4 T cells themselves: hyporesponses to IL-12, hyperresponses to IL-4, decreased expression of CD40 ligand. These factors contribute to the preferential production of $\mathrm{IL}-4, \mathrm{IL}-5$ and $\mathrm{IL}-10$, reflected by enhancement of IgGrand IgE antibodies and eosinophilia. In contrast, the low production of tumor necrosis factor- $\alpha$ (TNF- $\alpha$ ), IFN- $\gamma$ and weak generation of CD8 cytotoxic T lymphocytes (CTL) weakens the neonatal capacity to eliminate microorganisms and infected cells

microenvironment at the time of immune response triggering. As an example, reduced interleukin (IL)-12 production by activated mononuclear cells could be an important factor limiting NK cell activity since exogenous $\mathrm{L}-12$ was shown to enhance both IFN- $\gamma$ and NK lytic activity of cord blood human NK cells up to adult levels [13]. Inversely, reduced IFN- $\gamma$ production by NK cells probably affects the development of specific $\mathrm{T}$ cell responses, further contributing to the failure to generate the strong cellular responses required for clearance of intracellular microorganisms.

Antibody responses can be raised by newborns and infants in response to infectious agents or vaccines, although IgG responses are often slower and weaker than those elicited later in life. A lower avidity of vaccine-specific antibodies has also been reported, probably linked to poor T cell-driven affinity maturation of antibodies. This is best reflected by the additional doses of vaccines that must be given to fully immunize a 2-month-old infant compared to a 2-year-old toddler. Most importantly, responses to carbohydrate determinants such as those contained in bacterial capsules or in heavily glycosylated viral proteins can not be elicited in infants before 18-24 months of age. This does not relate to the capacity of $\mathbf{B}$ cells to recognize the antigen, and the basis for these impaired responses to carbohydrates - which explain infant susceptibility to encapsulated bacteria - remain largely unknown.

In vitro studies of neonatal $\mathrm{T}$ cells have indicated a low frequency of $\mathrm{T}$ cell precursors, poor responses to anti-CD2 and anti-CD3 stimulation in absence of anti-CD28 
co-stimulation, poor B cell help in terms of both cytokine production and CD40 ligand expression by activated $\mathrm{T}$ cells, and low cytokine production (mainly IL-2 and IFN- $\gamma$ ). Recently, neonatal T cells have been shown to follow a preferential development into IL-4- and IL-5-producing effector cells $[8,28]$ and to respond to specific cytokines such as IL-12 not only by IFN- $\gamma$ but also by IL-4 production [24].

Similar observations have been generated in vivo, in murine models of neonatal immunization with conventional vaccines. When the capacity of BALB/c mice at different stages of immunological maturation to respond to a selection of vaccine antigens and presentation systems was assessed, significant B and T cell responses to vaccine antigens (tetanus and MV peptides, tetanus toxoid, live viral attenuated MV, canarypox recombinant measles vector or BCG) were obtained already in the 1st week of life. However, these neonatal responses differed qualitatively from adult responses by a decreased $\operatorname{IgG} 2 \mathrm{a} / \operatorname{IgG} 1$ ratio of vaccine-specific antibodies, the secretion of significantly higher IL-5 and lower IFN- $\gamma$ levels by vaccine-specific T cells and an impaired induction of cytotoxic $\mathrm{T}$ cell precursors [3]. This was interpreted as reflecting a biased pattern of Th2 versus Th1 responses induced upon early exposure to vaccines, and considered to reflect the situation occurring in human infants (Fig. 1). The polarization of T cells is believed to depend essentially on the APC capacity; antigen presentation by APC that do not express adequate levels of co-stimulators is indeed known to result in a selective Th2 activation [5]. Thus, the impaired development of Th1 neonatal responses to antigens, which directly contributes to their susceptibility to intracellular agents, could reflect defective neonatal APC-T cell interactions.

\section{Which vaccines are required for immunization in early life?}

The factors which limit infant responses to infectious agents also affect immune responses to vaccine antigens and result in an age-dependent immunogenicity of vaccines. Bacterial polysaccharide vaccines are not immunogenic before the age of 18-24 months, and although protein/conjugate vaccines can be offered already at a few weeks of age, their use in young infants requires the administration of additional vaccine doses to reach sufficient immunogenicity. Live viral or bacterial vaccines which are capable of inducing stronger cellular immune responses than subunit vaccines can often not be used in very early life; with a few exceptions, their early use is limited either by the persistence of antibodies of maternal origin or by safety concerns.

Thus, specific requirements have to be met by vaccines for them to be considered for immunization in early life. Candidate vaccines should induce protective vaccine responses against bacterial/viral pathogens to which infants are exposed early in life and represent a significant risk of either severe acute disease or persistent infections. These antibody/cellular responses should be inducible at a young age and confer protection before age of exposure to these specific microorganisms. Vaccine-induced immune responses should ideally be sufficiently sustained for long-term protection. In addition, novel vaccine candidates should avoid the induction of neonatal tolerance and induce infant vaccine responses in spite of the persistence of antibodies of maternal origin. Finally, specific vaccine safety issues associated with this early age of relative immunodeficiency have to be met. The purpose of this review is to evaluate how DNA vaccines compare to conventional vaccines for their potential use in early life, based on the experimental observations generated so far in murine models of immunization. 


\section{DNA vaccines induce vaccine responses upon neonatal immunization}

Optimal vaccine candidates for early life immunization should be more rapidly protective than has been achieved so far with conventional vaccines. This depends on both the earliest age at which immune responses can first be triggered and the kinetics of the development of such responses.

Experiments on neonatal DNA immunization have been initiated in mice, and are currently being extended to primates. The capacity of vaccines to induce specific responses at various stages of the immune maturation was assessed by immunizing mice intramuscularly prior to 3 weeks of age or as adults. Conventional and DNA vaccines were selected in view of their potential clinical significance to target potential infant pathogens such as measles \{attenuated MV, immunodominant peptides of MV hemagglutinin (MV-HA), live recombinant canarypox (ALVAC-HA [26]) or DNA (MV-HA DNA [6]) vectors encoding MV-HA\} and tetanus \{tetanus toxoid (TT), immunodominant peptides of TT or DNA plasmid encoding its fragment C (TetC DNA [1])\}.

\section{Antibody responses}

Characteristics of vaccine antibodies induced in both age groups by either conventional [3] or DNA [14] vaccines are summarized in Table 1. As a rule, conventional vaccines resulted in weaker primary antibody responses when used in early life than in adult mice, although antibody titers often reached similar antibody titers after administration of a booster immunization. In contrast, a single injection of DNA vaccines performed in adult or young mice resulted 4 weeks later in similar antibody titers in both age groups. These titers were in general lower than those raised in adult mice by a single dose of conventional vaccines. Administration of a second DNA immunization similarly boosted antibody levels (tenfold) in both age groups.

Analysis of vaccine-specific antibody isotypes induced by conventional vaccines revealed an age-dependent limitation of the induction of IgG2a vaccine-specific antibodies by neonatal immunization even in situations where similar titers of IgG1 responses were induced (Table 1) [3]. This was also true for live vaccines such as ALVAC-HA. In contrast, similar antibody patterns were induced by early or adult DNA immunization, characterized by a preferential IgG2a (MV-HA) or a mixed IgG1/IgG2a (TetC DNA) profile of vaccine-specific antibodies [14]. Thus, in contrast to conventional vaccines, the use of DNA vaccines was capable of inducing adult-like neonatal antibody responses, both quantitatively and qualitatively.

\section{CD4 $T$ cell responses}

Comparison of $\mathrm{CD} 4 \mathrm{~T}$ cell responses induced by adult/neonatal immunization with conventional vaccines revealed quantitatively similar $T$ cell proliferation but significant qualitative differences (Table 2). The pattern of CD4 responses observed after immunization of 1- to 3-week-old mice with conventional vaccines consisted of a significantly higher production of IL-5 by antigen-specific $\mathrm{T}$ cells, as compared to cells from adult primed mice [3, 4]. This neonatal burst of IL-5 was mostly observed after neonatal immunization with alum-adsorbed subunit vaccines. It also occurred, however, upon immunization with live viral vaccines (live attenuated MV or ALVAC-HA), 
Table 1. Vaccine-specific antibodies induced by conventional and DNA vaccines ${ }^{\mathrm{a}}$

\begin{tabular}{|c|c|c|c|c|c|c|}
\hline \multirow[t]{3}{*}{ Vaccine types } & \multirow[t]{3}{*}{ Vaccines } & \multirow[t]{3}{*}{ Vaccine responses } & \multicolumn{4}{|c|}{ Serum antibody titers (ELISA- $\log 10$ ) } \\
\hline & & & \multicolumn{2}{|c|}{ Adult priming } & \multicolumn{2}{|c|}{ Neonatal priming } \\
\hline & & & $\operatorname{IgG}$ & $\operatorname{IgG} 2 a$ & $\operatorname{IgG}$ & $\operatorname{IgG} 2 a$ \\
\hline \multicolumn{7}{|l|}{ Measles } \\
\hline \multirow[t]{2}{*}{ Peptide } & MVH4972 & Primary & 2.2 & $<1.6$ & 1.7 & $<1.6$ \\
\hline & MVH4972 & Secondary & 3.8 & 1.8 & $1.9 *$ & $<1.6$ \\
\hline \multirow[t]{2}{*}{ Atten. virus } & MVSchwartz & Primary & 5.5 & 4.0 & $4.7^{*}$ & $2.3^{*}$ \\
\hline & MVSchwartz & Secondary & 6.8 & 4.2 & 6.8 & $2.3^{*}$ \\
\hline \multirow[t]{2}{*}{ Avipox vect. } & ALVAC-HA & Primary & 5.4 & 4.2 & $4.3^{*}$ & $2.1^{*}$ \\
\hline & ALVAC-HA & Secondary & 6.3 & 4.7 & 6.4 & $2.3 *$ \\
\hline \multirow[t]{2}{*}{ DNA racc. } & MV-HA DNA & Primary & 4.2 & 3.0 & 4.1 & 3.3 \\
\hline & MV-HA DNA & Secondary & 5.1 & 3.3 & 5.2 & 3.6 \\
\hline \multicolumn{7}{|l|}{ Tetanus } \\
\hline \multirow[t]{2}{*}{ Peptide } & ТTP30 & Primary & 3.7 & $<1.6$ & $2.5 *$ & $<1.6$ \\
\hline & ТТР30 & Secondary & 5.5 & 3.5 & 5.1 & $1.8 *$ \\
\hline \multirow[t]{2}{*}{ Protein } & TT & Primary & 6.3 & 4.2 & $5.2^{*}$ & $2.9^{*}$ \\
\hline & $\mathrm{TT}$ & Secondary & 7.2 & 5.0 & 6.6 & $3.4^{*}$ \\
\hline DNA vacc. & TetC DNA & Primary & 4.3 & 3.9 & 4.2 & 3.6 \\
\hline
\end{tabular}

${ }^{a}$ Antibody titers to measles virus hemagglutinin (MV-HA) or tetanus toxoid (TT) were measured by an enzyme-linked immunosorbent assay (ELISA) as described [3]. Analyses were performed in the serum after one or two immunizations of $\mathrm{BALB} / \mathrm{c}$ mice primed either as adults or at 1 week of age with the following peptide (MVH4972: $100 \mu \mathrm{g}$ in ALOH; TTP30: $100 \mu \mathrm{g}$ in ALOH), protein (TT: $3 \mu \mathrm{g}$ in ALOH), viral (MVSchwartz: $5 \times$ $10^{6} \mathrm{CCID} 50$ in ALOH; ALVAC-HA: $5 \times 10^{7} \mathrm{pfu}$ ) or DNA (MV-HA DNA: $100 \mu \mathrm{g}$; TetC DNA: $100 \mu \mathrm{g}$ ) vaccines $* P<0.05$ between adult and neonatal responses, Wilcoxon rank test

which furthermore failed to induce in young mice adult levels of IFN- $\gamma$ production by antigen-specific T cells.

These experiments, as well as those reported by others $[9,18,19,25]$, indicated that the impairment of neonatal cellular responses could derive from a preferential polarization of neonatal CD4 T cells towards a Th2 rather than a Th1 pattern, as reflected by the generation of antibodies of different isotypes (preferential IgG1 and IgE vs. IgG $2 \mathrm{a}$ antibodies), by a low secretion of IFN $\gamma$ and by an excess of IL-5 production by antigen-specific CD4 T cells. Although the distinction between Th1/Th2 subsets is less stringent in humans, a tendency towards preferential $\mathrm{Th} 2$ development of neonatal responses has also been observed in a few studies involving human cells [8, 28]. It could account for the age-dependent clinical pattern of infectious diseases such as hepatitis, mononucleosis or toxoplasmosis in which symptoms are believed to be essentially immune mediated and which remain mostly asymptomatic in young children compared to adults. It would also explain the lower immunogenicity and higher frequency of complications following BCG immunization performed at birth rather than at a few months of age $[11,16]$. Finally, it could be linked to the preferential induction of allergic reactions upon early exposure to environmental antigens.

The capacity of DNA vaccines to induce strong Th1 cellular responses in adult animals suggested that this novel antigen-presentation system could represent an inter- 
Table 2. Antigen-specific cytokine production by $\mathrm{T}$ cells induced by conventional and DNA vaccines ${ }^{\mathrm{a}}$

\begin{tabular}{|c|c|c|c|c|c|c|}
\hline \multirow[t]{3}{*}{ Vaccine types } & \multirow[t]{3}{*}{ Vaccines } & \multirow{3}{*}{$\begin{array}{l}\text { Vaccine } \\
\text { responses }\end{array}$} & \multicolumn{4}{|c|}{ Antibody-specifc cytokines } \\
\hline & & & \multicolumn{2}{|l|}{ IFN- $\gamma$} & \multicolumn{2}{|l|}{$\Pi \mathrm{L}-5$} \\
\hline & & & Adult & Neonatal & Adult & Neonatal \\
\hline \multicolumn{7}{|l|}{ Measles } \\
\hline Peptide & MVH4972 & Primary & $<20$ & $<20$ & 383 & $1310^{*}$ \\
\hline Atten. virus & MVSchwartz & Primary & 230 & $100^{*}$ & 500 & $2150^{*}$ \\
\hline Avipox vect. & ALVAC-HA & Primary & 150 & $52 *$ & $<150$ & $925^{*}$ \\
\hline DNA vacc. & MV-HA DNA & Primary & 182 & 315 & $<150$ & $<150$ \\
\hline \multicolumn{7}{|l|}{ Tetanus } \\
\hline Peptide & TTP30 & Primary & $<20$ & $<20$ & $<150$ & $800^{*}$ \\
\hline Protein & TT & Primary & $<20$ & $<20$ & $<150$ & $1305^{*}$ \\
\hline DNA vacc. & TetC DNA & Primary & 170 & 95 & 400 & 603 \\
\hline
\end{tabular}

${ }^{a}$ Cytokine production by antigen-specific T cells was measured by ELISA as described [3] in the supernatant of splenocytes after in vitro restimulation with MV or TT. Analyses were performed after a single immunization of BALB/c mice primed either as adults or at 1 week of age with the following peptide (MVH4972: $100 \mu \mathrm{g}$ in ALOH; TTP30: $100 \mu \mathrm{g}$ in ALOH), protein (TT: $3 \mu \mathrm{g}$ in ALOH), viral (MVSchwartz: $5 \times 10^{6}$ CCID50 in ALOH; ALVAC-HA: $5 \times 10^{7} \mathrm{pfu}$ ) or DNA (MV-HA DNA: $100 \mu \mathrm{g}$; TetC DNA: $100 \mu \mathrm{g}$ ) vaccines $* P<0.05$ between adult and neonatal responses, Wilcoxon rank test

esting vaccine strategy for neonatal immunization against intracellular agents. When CD4 T cell responses were assessed in mice immunized with DNA vaccines either as adults or at 1 week of age [14], similar levels of antigen-specific $T$ cell proliferation were observed. Importantly, the cytokine profiles of in vitro-restimulated splenocytes were similar in both age groups (Table 2). Depending on the antigen, either a preferential secretion of IFN- $\gamma$ (MV-HA) or mixed IFN- $\gamma$ and IL-5 production (tetanus fragment $\mathrm{C}$ ) was observed after DNA immunization. These patterns were directly associated with either the preferential $\operatorname{IgG} 2 \mathrm{a}$ or the mixed $\mathrm{IgG} 1 / \operatorname{IgG} 2$ a profile of vaccine-specific antibodies, respectively. They demonstrate the induction of Th0/Th1 responses upon neonatal DNA immunization. This property of DNA vaccines to induce strong Th1 responses in early life appears quite unique: it is apparently only shared with low doses of live replicating viral agents ([19] and unpublished observations) and suggests the existence of specific requirements for complete activation of neonatal versus adult Th1 responses. Our working hypothesis is that activation of neonatal dendritic cells requires a prolonged exposure to antigen, a feature shared by both DNA and live replicating infectious agents or vaccines, to fully trigger NK cell and Th1 responses.

\section{CD8 cytotoxic responses}

Among conventional vaccines, only live viruses are capable of raising significant cytotoxic T lymphocyte (CTL) responses in immunologically mature animals or humans. However, these responses are weakly induced by neonatal immunization. As an example, adult-like murine CTL responses can not be induced before the age of 3 weeks even with live vaccines such as ALVAC-HA, which raise very strong CTL responses in adult animals: a greater than tenfold reduction of the frequency of CTL precursors 
(CTLp) was observed upon immunization of 1- to 3-week-old versus adult-primed animals [3]. Although CTL can be induced in infants by infectious agents such as HIV or RSV, the limited generation of CTL responses in very early life is considered to participate in the impaired clearance of intracellular microorganisms. Furthermore, neonatal use of live vaccines is limited by both the relative physiological immunodeficiency and the presence of antibodies of matemal origin which limit the in vivo replication required by attenuated vaccines to reach sufficient immunogenicity.

DNA vaccines have emerged as potent inducers of CD8 cytotoxic responses in adult animals. This was found to be also the case upon neonatal DNA immunization. In contrast to the limited induction of neonatal CTL by ALVAC-HA, we observed the induction of adult-like CTL responses (assessed by similar frequency of CTLp in spleens) in $\mathrm{BALB} / \mathrm{c}$ mice vaccinated either in the neonatal period or as adults with MV-HA DNA [14]. The same observation was made in C57BL/6 mice immunized either as adults or in the first weeks of life with a DNA plasmid encoding the nucleoprotein of another paramyxovirus, the Sendai virus [14]. Successful induction of CTL by DNA immunization was reported by Sarzotti et al. [20] in yet another mouse strain: 2-day-old NFS/N mice raised a protective CD8-mediated CTL response upon intramuscular immunization with a DNA plasmid encoding the full-length genome of a neuropathogenic retrovirus (Cas-Br-M murine leukemia virus). Thus, the generic property of DNA vaccines to induce strong CTL responses, which has been linked to the presence in bacterial DNA of specific unmethylated CpG motives capable of rapidly enhancing transcription of IL-6, IFN- $\alpha$, IFN- $\gamma$ and IL-12 in APC $[2,12,21]$, is also effective in newborns. Through their adjuvant properties, DNA vaccines can fully activate neonatal dendritic cells, sharing only with live replicating agents the capacity to induce the adult-like neonatal CD8 responses required for clearance of intracellular microorganisms.

\section{How rapidly do immune responses to neonatal DNA immunization develop?}

In these murine models of neonatal immunization involving several mouse strains, plasmid vectors and antigens, a single DNA immunization performed in early life was capable of generating adult-like vaccine responses even in situations where conventional vaccines with the corresponding antigens failed to do so. However, the kinetics of vaccine response induced by DNA immunization was rather slow (2-6 weeks) in both adult and neonatally primed animals. This slow induction affected antibody responses, contrasting with the more rapid induction (1-2 weeks) of responses by corresponding conventional vaccines. Similarly, CTL induction was only detected in all mice at 8 weeks post DNA immunization [20], although occurring earlier (4-5 weeks) in some animals. This rather slow kinetic of DNA-induced vaccine responses appears as a generic property of DNA immunization which could represent a disadvantage for the induction of early infant protection. This is especially important in view of the difficulty in reverting neonatally induced Th2 responses towards Th1 responses. This could not be achieved either by Th1-driving adjuvants [4] or by DNA immunization [14] given at adult age after neonatal priming with conventional vaccines. 


\section{Does neonatal DNA immunization carry the risk of inducing tolerance rather than immunity?}

Neonatal exposure to antigen under specific conditions has been shown in various rodent models to induce tolerance rather than immunity to the specific antigen. This was shown to depend on several factors such as the nature and dose of antigen and the timing of exposure, which usually has to occur within the first $24 \mathrm{~h}$ of life. Contradictory reports on the risk of inducing neonatal tolerance by DNA immunization have been generated to date. When newborn BALB/c mice were immunized once within their first 24 h of life with MV-HA DNA, we observed the induction of strong immune responses characterized by similar antibody titers, IFN- $\gamma$ production and high frequency of CTLp as compared to 1-week-old or adult-primed mice. In another model, a single immunization of newborn $\mathrm{C} 3 \mathrm{H} / \mathrm{He}$ mice with a plasmid vector expressing the rabies virus glycoprotein was capable of raising significant antibody responses [29]. Neonatal priming was confirmed by booster administration, and antibody isotypes and cytokine production were reported as similar to those observed after adult immunization. In contrast to these results, a DNA vaccine encoding the circumsporozoite protein (CSP) of the Plasmodium yoelii malaria parasite was described as inducing long-lasting tolerance in 2-day- to 1-week-old mice who failed to raise immune responses upon adult boosting with DNA [15]. The mechanisms underlying this described tolerance induction are uncertain. They could be linked to unique properties of this CSP-DNA construct, which mainly induces Th2 rather than Th0/Th1 responses in adult mice [22] and whose immunogenicity clearly differs from the native CSP protein in terms of antigenic determinants [15]. Until further information is available, the induction of immune tolerance by DNA immunization of newborn mice with certain DNA plasmids can thus not be excluded. The importance of this specific observation might, however, not be directly relevant to human infant immunization, since the maturation of their immune system at birth is such that tolerance induction has not yet been induced by postnatal exposure to any antigen. Thus, neonatal DNA immunization appears worth assessing in conditions where induction of early immune responses are desired.

\section{Can sustained vaccine responses be induced by neonatal DNA immunization?}

Infant immunization against hepatitis B would be of little interest if protection waned before adolescence and its risk of exposure through sexual transmission. Waning of immunity in the absence of a booster is commonly observed with conventional vaccines such as inactivated or subunit vaccines, and there is little evidence yet that even live attenuated vaccines would provide life-long immunity in the absence of a repeat exposure to wild-type infectious agents. Interestingly, a single DNA immunization was shown in adult mice to induce long-lasting responses and life-long maintenance of memory cells to various antigens. Prolonged immune responses are similarly observed after neonatal DNA immunization [7, 27], and this could represent a significant advantage of DNA vaccines over conventional vaccines. However, experiments performed in non-human adult primates are now showing transient immune responses which suggest that this feature could be essentially a characteristic of rodent responses to DNA immunization. A weak and transient production of antibodies was also observed in a first study assessing DNA immunization of newborn chimpanzees against hepatitis B virus [17]. However, in spite of negative or very low antibody titers, the 
two animals were protected from a viral challenge performed at 33 weeks, suggesting the successful induction of cell-mediated responses.

\section{Can vaccine responses be induced by neonatal DNA immunization in spite of the persistence of antibodies of maternal origin?}

A number of infectious diseases can be either prevented or attenuated by the passive transfer of specific antibodies. Prevention of neonatal infections also relies on transplacental transfer of antibodies of maternal origin from mother to child. These antibodies progressively decline below the protective level, but nevertheless remain capable for a period of time of interfering with infant responses to immunization. The precise mechanisms responsible for the variable effects of maternal antibodies on infant responses have not yet been fully deciphered. It is known, however, that live attenuated vaccines have the greatest susceptibility to the inhibitory influence of maternal antibodies: by neutralizing part of the vaccine inoculum prior to viral entrance within host cells, antibodies interfere with the replication cycle in vivo that it requires to induce protective responses. This is of significant clinical importance for measles immunization in countries where vaccine coverage is not yet sufficient to limit the risk of contracting measles prior to the age at which maternal antibodies have sufficiently declined in most infants to allow successful immunization [23].

To assess the influence of MV-specific maternal antibodies on neonatal responses to conventional or DNA vaccines expressing measles hemagglutinin, 2-week-old mice born of nonimmune mothers were passively transferred various concentrations of syngeneic immune serum raised by two immunizations with live attenuated measles vaccine. They were immunized 2 days later with either live attenuated measles vaccine, live recombinant ALVAC-HA or MV-HA DNA, and followed at regular intervals for determination of HA-specific antibodies. When immunization was performed in presence of HA-specific antibodies at titers corresponding to those observed in pups of immune mothers $(>4.5 \log 10)$, neither of the live vaccines nor DNA was capable of inducing HA-specific antibodies in the pups (manuscript in preparation). When concentrations of passively transferred antibodies were tenfold lower at time of immunization, both conventional and DNA vaccines were capable of inducing infant antibody responses. Thus, DNA vaccines could be reported either as capable or not of circumventing the inhibitory influence of maternal antibody, depending on the antibody titers present at time of immunization. This suggests that passive antibodies bind to a fraction of the antigen expressed by transfected muscle cells which is sufficient, or not, to prevent the induction of antibody responses. In this model of measles immunization, DNA vaccines, therefore, did not show any advantage over live conventional vaccines for infant immunization in the presence of passively transferred antibodies.

\section{Does neonatal DNA immunization have to meet specific safety issues?}

Ongoing human trials of DNA immunization suggest that these vaccines have no immediate or early side effects. However, nothing is known yet on the potential longterm consequences of such vaccines. The issues of prolonged antigen expression being potentially capable of triggering physiopathological immune responses and the questions of potential genomic integration will have to be specifically addressed in neona- 
tal models of immunization, bearing in mind the ongoing maturation of the immune system and the high mitotic rates existing in infants.

\section{Conclusion}

DNA vaccines favorably compare to conventional vaccines in their unique capacity to induce, in murine models, adult-like antibody, Thl and CTL responses at a time of yet significant immune immaturity. Immune responses to DNA vaccines are, however, significantly slower than the one induced by adjuvanted subunit or live vaccines. Although they persist for life in mice, preliminary data in non-human adult primates suggest that this could not be the case in higher mammals. The issue of potential tolerance induction will likely not emerge as a critical feature of human neonatal DNA immunization. However, DNA vaccines are unlikely to prove superior to conventional vaccines in their capacity to circumvent the inhibitory influence of maternal antibodies.

Thus, the greater perspectives for neonatal DNA immunization could be found in models where the induction of Th1 and CTL responses are of utmost importance. These are essentially infections with intracellular agents responsible for severe/persistent infections upon early exposure and for which no current efficient and safe conventional vaccine exists. Evaluating neonatal DNA immunization strategies against RSV or herpes viruses, tuberculosis or Chlamydiae therefore emerge as sound priorities.

Acknowledgements. Our work is supported by grants from the Swiss National Science Foundation, from W.H.O. Global Programme for Vaccines and Immunization, from Sandoz and from Pasteur-Mérieux Serums et Vaccins. C.A. Siegrist is supported by the Swiss National Science Foundation (SCORE A).

\section{References}

1. Anderson R, Gao SM, Papakonstantinopoulou A, Roberts M, Dougan G (1996) Immune response in mice following immunization with DNA encoding fragment $\mathrm{C}$ of tetanus toxin. Infect Immun 64:3168

2. Ballas ZK, Ramuaawn WL, Krieg AM (1996) Induction of NK activity in murine and human cells by CpG motifs in oligodeoxynucleotides and bacterial DNA. J Immunol 157:1840

3. Barrios C, Brawand P, Berney M, Brandt C, Lambert PH, Siegrist CA (1996) Neonatal and early life immune responses to various forms of vaccine antigens qualitatively differ from adult responses: predominance of a Th2-biased pattern which persists after adult boosting. Eur J Immunol 26: 1489

4. Barrios C, Brandt C, Berney M, Lambert PH, Siegrist CA (1996) Partial correction of the Th2/Th1 imbalance in neonatal murine responses to vaccine antignes through selective adjuvant effects. Eur $\mathbf{J}$ Immunol 26:2666

5. Burstein HJ, Shea CM, Abbas AK (1992) Aqueous antigens induce in vivo tolerance selectively in IL-2 and IFN-gamma producing Th1 cells. J Immunonol 148:3687

6. Cardoso AI, Blixenkrone-Moller M, Fayolle J, Liu M, Buckland R, Wild TF (1996) Immunization with plasmid DNA encoding for the measles virus hemagglutinin and nucleoprotein leads to humoral and cell-mediated immunity. Virology 225:293

7. Davis HL, Michel M, Whalen RG (1993) DNA-based immunization for hepatitis B induces continuous secretion of antigen and high levels of circulating antibody. Hum Mol Genet 2:1847

8. Demeure CE, Yang LP, Byun DG, Ishihara H, Vezzio N, Delespesse G (1995) Human naive CD4 T cells produce interleukin-4 at priming and acquire a $\mathrm{Th} 2$ phenotype upon repetitive stimulation in neutral conditions. Eur J Immunol 25:2722

9. Forsthuber T, Yip HC, Lehman PV (1996) Induction of TH1 and TH2 immunity in neonatal mice. Science $271: 1728$ 
10. Hunt DW, Huppertz HI, Jiang HJ, Petty RE (1994) Studies of human cord blood dendritic cells: evidence for functional immaturity. Blood 84:4333

11. Ildirim I, Sapan N, Cavusoglu B (1992) Comparison of BCG vaccination at birth and at third month of life. Arch Dis Child 67:80

12. Klinman DM, Yi AK, Beaucage SL, Conover J, Krieg AM (1996) CpG motifs present in bacterial DNA rapidly induce lymphocytes to secrete interleukin 6 , interleukin 12 and interferon $\gamma$. Proc Natl Acad Sci USA 93:2879

13. Lee SM, Suen Y, Chang L, Bruner V, Quian J, Indes J, Knoppel E, Ven C van de, Cairo MS (1996) Decreased interleukin-12 (IL-12) from activated cord versus adult peripheral blood mononuclear cells and upregulation of interferon-gamma, natural killer and lymphokine-activated killer activity by IL-12 in cord blood. Blood 88:945

14. Martinez X, Brandt C, Saddallah F, Tougne C, Barrios C, Wild F, Dougan G, Lambert PH, Siegrist CA (1997) DNA immunization circumvents deficient induction of TH1 and CTL responses in neonates and during early life. Proc Natl Acad Sci USA (in press)

15. Mor G, Yamschchikov G, Sedegah M, Takeno M, Wang R, Houghten RA, Hoffman S, Klinman DM (1996) Induction of neonatal tolerance by plasmid DNA vaccination of mice. J Clin Invest 98:2700

16. Pabst HF, Godel JC, Spady DW, McKechnie J, Grace M (1989) Prospective trial of timing of BCG vaccination in Canadian Cree infants. Am Rev Respir Dis 140:1007

17. Prince AM, Whalen R, Brotman B (1997) Successful nucleic acid based immunization of newborn chimpanzees against hepatitis $B$ virus. Vaccine (in press)

18. Ridge JP, Fuchs EJ, Matzinger $P$ (1996) Neonatal tolerance revisited: tuming on newborn $T$ cells with dendritic cells. Science 271:1723

19. Sarzotti M, Robbins DS, Hoffman PM (1996) Induction of protective CTL responses in newborn mice by a murine retrovirus. Science 271:1726

20. Sarzotti M, Dean TA, Remington MP, Ly CD, Furth PA, Robbins DS (1997) Induction of CTL responses in newborn mice by DNA immunization. Vaccine (in press)

21. Sato Y, Roman M, Tighe H, Lee D, Corr M, Nguyen MD, Silverman GJ, Lotz M, Carson DA, Raz E (1996) Immunostimulatory DNA sequences necessary for effective intradermal gene immunization. Science 273:352

22. Sedegah M, Hedstrom R, Hobart P, Hoffman SL (1994) Protection against malaria by immunization with plasmid DNA encoding circumsporozoite protein. Proc Natl Acad Sci USA 90:4156

23. Shasby M, Shope T, Downs H, Herrmann KL, Polkowsky J (1977) Epidemic measles in a highly vaccinated population. N Engl J Med 296:585

24. Shu U, Demeure CE, Byun DG, Podlaski F, Stern AS, Delespesse G (1994) Interleukin 12 exerts a differential effect on the maturation of neonatal and adult human CD45RO- CD4 T cells. J Clin Invest 94:1352

25. Singh RR, Hahn BH, Sercarz EE (1996) Neonatal peptide exposure can prime T cells and, upon subsequent immunization, induce their immune deviation: implications for antibody vs. T cell mediated autoimmunity. J Exp Med 183:1613

26. Taylor J, Pincus S, Tartaglia J, Richardson C, Alkhatib G, Briedis D, Appel M, Norton E, Paoletti E (1991) Vaccinia virus recombinants expressing either the measles virus fusion or hemagglutinin glycoprotein protect dogs against canine distemper virus challenge. J Virol 65:4263

27. Yakaucas MA, MorrowJE, Parker SE, Abai A, Rhodes GH, Dwarki VJ, Gromkowski SH (1993) Longterm anti-nucleoprotein cellular and humoral immunity is induced by intramuscular injection of plasmid DNA containing NP gene. DNA Cell Biol 12:771

28. Yang LP, Byun DG, Demeure CE, Vezzio N, Delespesse G (1996) Default development of cloned human naive CD4 T cells into interleukin-4- and interleukin-5-producing effector cells. Eur J Immunol 25:3517

29. Wang, Y, Xiang Z, Pasquini S, Ertl HCJ (1997) Immune response to neonatal genetic immunization. Virology $228: 278$ 